\title{
Nonlocal scalar field in an external potential: The WKB approximation
}

\author{
Valeri P. Frolov $\odot^{*}$ \\ Theoretical Physics Institute, University of Alberta, Edmonton, Alberta T6G 2E1, Canada
}

(Received 23 November 2020; accepted 17 December 2020; published 20 January 2021)

\begin{abstract}
We consider a nonlocal theory of a scalar massive field in a flat spacetime background in the presence of an external potential and construct WKB solutions for this theory. We use a model in which the kinetic part of the scalar field action is modified by changing $\square$ to $\square f(\square)$ operator. We discuss conditions when the corresponding form factor $f$ is chosen so that the theory does not contain new unphysical degrees of freedom. We applied the obtained WKB solutions for study energy levels of the field trapped by a onedimensional potential and the probability of the barrier penetration. This allows us to illustrate how the effects of the nonlocality change the known results obtained for the local field theory.
\end{abstract}

DOI: 10.1103/PhysRevD.103.025020

\section{INTRODUCTION}

The idea of nonlocality is quite old in theoretical physics. Nonlocal modifications of the field theory were discussed already in the publications [1-3]. Even if one works with a local quantum field theory, already at the one loop level, its effective action is nonlocal. This nonlocality is connected with the vacuum polarization and particle creation effects, and it reflects the fact that a vacuum is a physical medium and in this sense it behaves as a condensed matter with very special properties. The nonlocality that we consider in this paper is of a different origin. We assume that a field theory is described by a nonlocal action already at the tree level. In particular this means that its interaction with external sources, as well as a self-interaction, is nonlocal.

Usually such a nonlocal modification contains a scale parameter which determines either the energy at which the effects of the nonlocality become important or the corresponding spacetime length when it happens. Nonlocal theories were discussed in the beginning of seventies of the past century by Efimov [4-7]. The nonlocality is often introduced to modify high energy properties of the theory and to improve its ultraviolet behavior. More recently, the interest in nonlocal theories increased. This was mainly stimulated by the development of the string theory. Nonlocal fields naturally arise in the string theory and in the theories with a noncommutative geometry (see e.g., [8-15] and references therein). Nonlocal effects in quantum gravity were widely discussed recently. A comprehensive

*vfrolov@ualberta.ca

Published by the American Physical Society under the terms of the Creative Commons Attribution 4.0 International license. Further distribution of this work must maintain attribution to the author(s) and the published article's title, journal citation, and DOI. Funded by SCOAP. review of modifications of gravity involving a minimal length scale and related references can be found in [16].

There is a subclass of the nonlocal field theories sometimes called ghost-free theories. An effective action in these models contains an infinite number of derivatives so that the corresponding field equations are effectively nonlocal. These models have been widely discussed recently, and they have rather "nice properties." In these theories the nonlocality is introduced in such a way that it preserves local Lorentz invariance, and it does not introduce new unphysical degrees of freedom (ghosts). In these models the ultraviolet (UV) behavior of the theory at short distances is improved, while in the infrared (IR) regime (at large scales) they reproduce results of a corresponding local theory [17-23]. The main motivation for the study of such infinite derivative modifications of the gravity equations is connected with attempts to solve long-standing problems of cosmological and black hole singularities [18,24-34].

Complete equations of a modified gravity, which include both nonlocality and nonlinearity, are quite complicated. It is much easier to study a linearized version of the theory. However, already the study of linearized models allows one to obtain several stimulating results: (i) The nonlocality removes singularities of the field produced by pointlike sources [18,35-38]; (ii) There exists a mass gap for mini black hole formation [39,40]; (iii) It allows one to demonstrate a formation of the inner horizon for black hole creation in the scattering of ultrarelativistic particles [41]. A standard technique for solving these problems is usage of nonlocal Green functions, which for the flat spacetime background can be found by means of the Fourier transform.

The study of the nonlocal field in the presence of an external potential is a much more complicated problem. There exists very special cases when it is possible to find an explicit solution. For example, such a solution was obtained 
for scattering of a nonlocal scalar field by a deltalike potential [42-44]. However, in a general case, in the presence of an arbitrary potential one needs to solve a nonlocal linear equation with the space-dependent coefficients, which is a very nontrivial problem.

The purpose of this paper is to demonstrate that one can obtain asymptotic solutions for this problem by using a standard WKB method. Its main idea is to search for a solution $\Phi(x)$ of the field equation in the form $\Phi(x) \approx u(x) \exp [i S(x) / \hbar]$. After substitution of this ansatz into the field equation, one collects terms of the expansion into the powers of $\hbar$ which are of the same order of $\hbar$. In the leading order one gets a first-order partial differential equation of the form $H(\nabla S, x)=0$ known as an eikonal equation. A subleading equation determines evolution of a slowly changing field amplitude $u(x)$. The eikonal equation can be identified with the Hamilton-Jacobi equation for the Hamiltonian obtained by the substitution of $\nabla S=p$ into it. Initial data for the Hamilton-Jacobi equation specify a beam of trajectories in the phase space which forms a Lagrangian submanifold (for details see e.g., a remarkable book [45]). Knowledge of this Lagrangian submanifold allows one not only to construct the eikonal function $S(x)$ but also to find a solution of the transport equation for the amplitude $u(x)$ by using the Liouville theorem. This WKB method is widely used in the standard quantum mechanics and field theory where the corresponding equations are second-order partial differential equations. However, it can be applied to a wider class of so-called quasilinear differential equations. A comprehensive presentation of these results can be found in the book [46]. In this paper we apply this method for study of quasiclassical solutions of the linear nonlocal scalar field equations in the presence of an external potential. A similar approach for other higher and infinite order equations can be found in [47].

This paper is organized as follows. In Sec. II we describe a model of a nonlocal scalar field with infinite number of derivatives, which is analyzed in the paper. In Sec. III we construct a WKB solution for such a field in the presence of an external potential in any number of spacetime dimensions. In Sec. IV we consider a special case when the potential depends on only one spatial Cartesian coordinate. Energy levels for the nonlocal scalar field confined by the one-dimensional parabolic potential are calculated in Sec. V. Under-barrier propagation of the nonlocal field and barrier penetration effect are discussed in Sec. VI. Section VII contains discussion of the obtained results. Additional technical details are collected in two appendices.

\section{NONLOCAL SCALAR FIELD EQUATION}

We consider $N$-dimensional flat spacetime. Its metric in Cartesian coordinates is

$$
d s^{2}=\eta_{\mu \nu} d x^{\mu} d x^{\nu}, \quad \mu, \nu=0,1, \ldots, \quad N-1,
$$

where $\eta_{\mu \nu}=\operatorname{diag}(-1,1, \ldots, 1)$. Let us consider a scalar massive field $\varphi$ obeying the Klein-Gordon equation

$$
\left(\hbar^{2} \square-m^{2}-V\right) \varphi=0 .
$$

Here $\square=\eta^{\mu \nu} \partial_{\mu} \partial_{\nu}, m$ is the mass of the field, and $V(x)$ is an external potential. In what follows we shall study solutions of this equation and its nonlocal generalization in the WKB approximation. For this reason we keep the Planck constant $\hbar$, while as usual put $c=1 .^{1}$ The KleinGordon equation follows from the action

$W[\varphi]=\frac{1}{2} \int d^{N} x \varphi(F(\square)-V) \varphi, \quad F(\square)=\hbar^{2} \square-m^{2}$.

We consider a generalization of the Klein-Gordon equation in which the operator $F(\square)$ is modified. Namely, we assume that it is a scalar operator which may contain an arbitrary (finite or infinite) number of partial derivatives $\partial / \partial x^{\mu}$. It is easy to check that the covariance of the action requires that such an operator can be written as a scalar function of the $\square$-operator. In order to keep a proper dimensionality of the action we introduce a parameter $\mu$, which has a dimension of the mass, and we write the operator $F(\square)$ in the form

$$
F(\square)=\mu^{2} \hat{F}\left(\hbar^{2} \square / \mu^{2}\right) .
$$

The corresponding generalized Klein-Gordon equation is

$$
[-\hat{F}(z)+\hat{V}] \varphi=0,
$$

where $z=\hbar^{2} \square / \mu^{2}$ and $\hat{V}=V / \mu^{2}$.

We consider a class of theories for which the function $\hat{F}(z)$ has the following properties:

(1) $\hat{F}(z)$ does not vanish anywhere on the complex plane of $z$ besides the point $z=\hat{m}^{2}$;

(2) $\left.\hat{F}\right|_{z=\hat{m}^{2}}=0$ and $d \hat{F}(z) /\left.d z\right|_{z=\hat{m}^{2}}=1$;

(3) For a real value of its argument $z$ the function $\hat{F}(z)$ is real.

These conditions guarantee that the inverse of the operator $\hat{F}$ has a single pole with its residue equal to 1 . In other words, there are no new unphysical (ghost) degrees of freedom in this theory. To satisfy the required properties it is sufficient to choose $\hat{F}$ in the form

$\hat{F}(z)=\mathcal{F}(\hat{z}), \quad \mathcal{F}(\hat{z})=\hat{z} f(\hat{z}), \quad \hat{z}=z-\hat{m}^{2}$,

where $f(\hat{z})=\exp (g(\hat{z}))$ and $g(\hat{z})$ is an entire function of the complex variable satisfying the condition $g(\hat{z}=0)=0$. We call $f(\hat{z})$ a form factor.

\footnotetext{
${ }^{1}$ In these units one has $[\hbar]=M L, \quad[\square]=L^{-2}$, and $\left[m^{2}\right]=[V]=M^{2}$.
} 


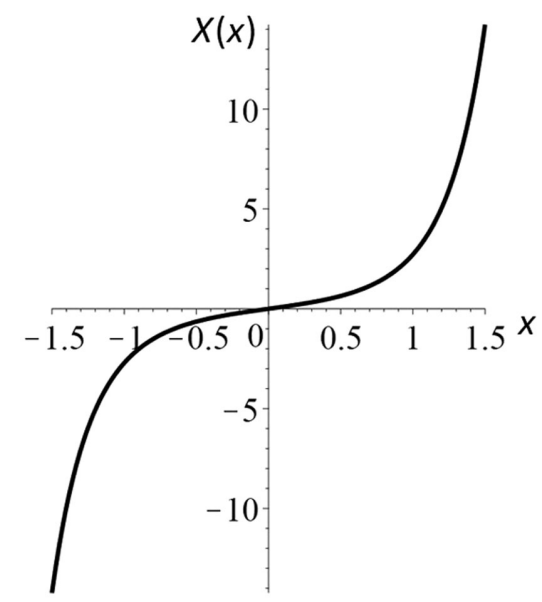

FIG. 1. Function $X(x)$.

In order to illustrate the results we shall use a special example of the form factor. Namely, we put $g(\hat{z})=\hat{z}^{2}$, then one has

$$
\mathcal{F}(\hat{z})=X(\hat{z}) \equiv \hat{z} \exp \left(\hat{z}^{2}\right)
$$

This function obeys all the conditions listed above. Besides this for real $\hat{z}$, it is a monotonically increasing from $-\infty$ at $\hat{z}=-\infty$ to $+\infty$ at $\hat{z}=+\infty$ and is positive for $\hat{z}>0$. One also has $\mathcal{F}(-\hat{z})=-\mathcal{F}(\hat{z})$. The function $X(x)$ is shown at Fig. 1. Other properties of this function, which are used later in the paper, can be found in Appendix B.

One can expand the function $\mathcal{F}(\hat{z})$ into powers of $\hat{z}$. This gives an infinite series of the powers of $\square$ for the field operator $\hat{F}\left(\hbar^{2} \square / \mu^{2}\right)$. This means that this operator is nonlocal. Let us also mention that the mass parameter $\mu$, which enters this operator, determines a characteristic energy scale at which the effects of the nonlocality become important. In the limit $\mu \rightarrow \infty$ the operator $F$ becomes $\hbar^{2} \square-m^{2}$, and the theory is local.

\section{WKB APPROXIMATION}

\section{A. A solution of the nonlocal field equation in the WKB approximation}

Let us write the nonlocal field Eq. (5) in the form ${ }^{2}$

$\hat{\mathcal{O}} \varphi=0, \quad \hat{\mathcal{O}}=\frac{1}{2}\left(-\mu^{2} \mathcal{F}(\hat{z})+\hat{V}\right), \quad \hat{z}=\hbar^{2} \square / \mu^{2}-\hat{m}^{2}$.

\footnotetext{
${ }^{2}$ In the nonlocal theory it is convenient to use the parameter $\mu$ to define dimensionless quantities. We specify such quantities by using a hat over them. Here and later we shall use the following notations

$$
\hat{m}=m / \mu, \quad \hat{\eta}=\eta / \mu, \quad \hat{\eta}_{0}=\eta_{0} / \mu, \quad \hat{V}=V / \mu, \quad \hat{z}=\hat{\eta}_{0}^{2}-\hat{\eta}^{2} .
$$
}

We include a factor $1 / 2$ in the operator $\hat{\mathcal{O}}$, which evidently does not change solutions but this simplifies the form of some relations. The operator $\hat{\mathcal{O}}$ belongs to the class of Eqs. (A1) discussed in the Appendix. We are looking for a solution of the field Eq. (8) in the WKB approximation and write it in the following form:

$$
\varphi(x)=\exp \left(\frac{i S(x)}{\hbar}\right) \sum_{j=0}^{\infty}\left(\frac{\hbar}{i}\right)^{j} u_{j}(x)
$$

Here $S(x)$ is a fast changing phase, while $u_{j}(x)$ are slowly changing functions. Let us emphasize that besides standard conditions of the validity of the WKB approximation formulated in textbooks on quantum mechanics, we also assume that the parameter of nonlocality $\mu$ does not depend on $\hbar$. We keep this parameter fixed in the limit $\hbar \rightarrow 0$. This allows us to discuss effects of the nonlocality in the quasiclassical approximation.

In order to find the eikonal function $S(x)$ and the amplitudes $u_{j}(x)$, we follow the steps described in Appendix A. First of all we define a symbol $H(x, p)$ of the operator $\hat{\mathcal{O}}$. Let $p_{\mu}$ be a covector and denote $p^{2}=p_{\mu} p^{\mu}$. Then one should substitute $-\hbar^{2} \square \rightarrow p^{2}$ into (8). The corresponding symbol for the operator $\hat{\mathcal{O}}$ is

$H(x, p)=\frac{1}{2}\left[-\mu^{2} \mathcal{F}(\hat{z})+V(x)\right], \quad \hat{z}=-\frac{1}{\mu^{2}}\left(p^{2}+m^{2}\right)$.

The eikonal Eq. (A8) implies that the following constraint $H(x, p)=0$ is valid.

The next step is to study a dynamical system in $2 N$ dimensional phase space satisfying Hamilton's equations

$$
\frac{d x^{\mu}}{d \tau}=\frac{\partial H}{\partial p_{\mu}}, \quad \frac{d p_{\mu}}{d \tau}=-\frac{\partial H}{\partial x^{\mu}} .
$$

Since the Hamiltonian $H(x, p)$ is an integral of motion, it is sufficient to choose $H(x, p)=0$ at the initial "time" $\tau=0$, then the constraint $H(x, p)=0$ is valid for any later time.

We define a $(N-1)$-dimensional surface $\Sigma$ by conditions

$$
x^{\mu}=f^{\mu}\left(y^{i}\right), \quad i=1, \ldots, N-1,
$$

and choose an initial state for the field $\varphi$ in the form

$$
\left.\varphi(x)\right|_{\Sigma}=u_{0}(y) \exp \left[\frac{i}{\hbar} S^{0}(y)\right] .
$$

After solving Eqs. (A13) we impose the initial conditions (A14) for the Hamiltonian Eqs. (11). Solving this system, we find 


$$
x^{\mu}=x^{\mu}\left(\tau, y^{i}\right), \quad p_{\mu}=p_{\mu}\left(\tau, y^{i}\right) .
$$

A sought solution $\varphi(x)$ of the nonlocal Eq. (8) in the leading order of the WKB approximation is

$\varphi(x)=u_{0}(y) \sqrt{\frac{J(0, y)}{J(\tau, y)}} \exp \left[\frac{i}{\hbar}\left(S^{0}(y)+\int_{0}^{\tau} p_{\mu} d x^{\mu}\right)\right]$.

This expression is obtained from (A19) by using the relation

$$
\frac{\partial^{2} H(x, p)}{\partial x^{\mu} \partial p_{\mu}}=0
$$

valid for the Hamiltonian (10). The integral in the exponent in (15) is taken over phase trajectories which belong to the Lagrangian submanifold determined by given initial conditions. Here the function $J$, which enters into the prefactor in (15), is defined by (A18).

\section{B. Remarks on the Hamilton's equations}

Let us discuss now the Hamilton's equations in more detail. One has

$$
\frac{\partial H}{\partial p_{\mu}}=\mathcal{F}^{\prime} p^{\mu}
$$

Here a prime denotes a derivative of $\mathcal{F}(\hat{z})$ with respect to its argument $\hat{z}$. Hence the Hamilton's Eqs. (11) take the form

$$
\begin{gathered}
\dot{x}^{\mu} \equiv \frac{d x^{\mu}}{d \tau}=\mathcal{F}^{\prime} p^{\mu}, \\
\dot{p}_{\mu} \equiv \frac{d p_{\mu}}{d \tau}=-\frac{1}{2} V_{, \mu} .
\end{gathered}
$$

The first of these equations implies

$$
\dot{x}^{2} \equiv \eta_{\mu \nu} \dot{x}^{\mu} \dot{x}^{\nu}=\left(\mathcal{F}^{\prime}\right)^{2} p^{2} .
$$

Since $\hat{z}=-\left(p^{2}+m^{2}\right) / \mu^{2}$, the right-hand side of this relation is a function of $p^{2}$. We assume that one can solve Eq. (20) and find $p^{2}$ as a function of $\dot{x}^{2}$. Then the Lagrangian of this dynamical system is

$$
\begin{aligned}
L(x, \dot{x}) & =\dot{x}^{\mu} p_{\mu}-H \\
& =\frac{1}{2}\left[\mu^{2}\left(\mathcal{F}-2 \hat{z} \mathcal{F}^{\prime}\right)-2 m^{2} \mathcal{F}^{\prime}\right]-\frac{1}{2} V(x) .
\end{aligned}
$$

It is understood that in this expression for $L$ one should express $\hat{z}$ as a function of $\dot{x}^{2}$. Since $L$ does not contain explicitly the parameter $\tau$, the corresponding "energy" for this Lagrangian is conserved. This energy is nothing but the Hamiltonian in which $p^{2}$ is a function of $\dot{x}^{2}$ defined by (20).
The constraint equation in the dimensionless form is (see footnote 2)

$$
-\mathcal{F}(\hat{z})+\hat{V}(x)=0
$$

If $\hat{V}(x) \geq 0$, then $\mathcal{F}(\hat{z})=\hat{z} f(\hat{z})$ should be real and positive. The argument of this function $\hat{z}=-\left(p^{2}+m^{2}\right) / \mu^{2}$ is real. Since $f(0)=1$ and $f(\hat{z})$ does not vanish, this function is positive for real $\hat{z}$. This means that the sign of $\mathcal{F}(\hat{z})$ coincides with the sign of $\hat{z}$. Equation (22) implies that the classical motion is possible only in a domain where $\hat{z} \geq 0$.

In the absence of the potential the constraint equation takes the form

$$
H_{0}(p)=-\frac{1}{2} \mu^{2} \hat{z} f(\hat{z})=0 .
$$

Since $f(\hat{z})$ does not vanish, this equation gives $p^{2}=-m^{2}$. The condition $\mu^{2} \mathcal{F}^{\prime}(\hat{z}=0)=1$ implies that

$$
\left.\frac{\partial H_{0}(p)}{\partial p_{\mu}}\right|_{p^{2}+m^{2}=0}=p^{\mu} .
$$

This means that in the absence of the potential the Hamilton's Eqs. (18) and (19) coincide with the equations for a free particle with the Hamiltonian $(1 / 2)\left(p^{2}+m^{2}\right)$. In other words, the effects of the nonlocality are important only off shell, that is, in the presence of the external potential.

\section{ONE-DIMENSIONAL CASE}

To illustrate an application of the general approach described in the previous section, we consider now a simple model. Namely, we assume that the external potential $V(x)$ depends on only one spatial Cartesian coordinate. We denote this coordinate by $q$ and use the following notations:

$$
x^{\mu}=\left(t, q, x_{\perp}\right), \quad x_{\perp}=\left(x^{2}, \ldots, x^{N}\right) .
$$

We use index $a=2, \ldots, N$ to enumerate transverse coordinates $x_{\perp}$. We write the eikonal function $S$, which enters the WKB solution (9), in the form

$S=-\varepsilon t+\left(p_{\perp}, x_{\perp}\right)+\mathcal{S}(q), \quad\left(p_{\perp}, x_{\perp}\right)=\sum_{a=2}^{N} p_{a} x^{a}$.

We also assume that the functions $u_{j}$ depend only on the coordinate $q$.

Let us assume first that the potential $V(q)$ vanishes. In order to solve the corresponding field Eq. (8) one can put $\mathcal{S}(q)=\eta q$ where $\eta=\mathrm{const}, u_{0}=\mathrm{const}$, and $u_{j \geq 1}=0$. This is a plane wave solution of the field Eq. (8). Denote $p_{\mu}=\left(-\varepsilon, \eta, p_{\perp}\right)$, where the components of the covector 
$p_{\perp}$ are $p_{a}$ with $a=2, \ldots, N$. Hence, $S=(p, x)=p_{\mu} x^{\mu}$. Other solutions of the field equation can be obtained by superimposing the plane-wave solutions, which in fact is just by using the Fourier representation. The constraint equation $p^{2}+m^{2}=0$ gives

$$
-\varepsilon^{2}+p_{\perp}^{2}+m^{2}+\eta^{2}=0 .
$$

In the presence of the potential we look for a solution of the nonlocal field Eq. (8) in the form

$$
\varphi(x)=\exp \left[\frac{i}{\hbar}\left(-\varepsilon t+\left(p_{\perp}, x_{\perp}\right)\right)\right] \Phi(q) .
$$

Substituting this expression into (8), one obtains the following reduced equation:

$$
\begin{gathered}
\hat{\mathcal{O}}_{1} \Phi=0, \\
\hat{\mathcal{O}}_{1}=\frac{1}{2}\left[-\mu^{2} \hat{D} f(\hat{D})+V(q)\right], \\
\hat{D}=\frac{1}{\mu^{2}}\left[\eta_{0}^{2}+\hbar^{2} \frac{\partial^{2}}{\partial q^{2}}\right], \quad \eta_{0}^{2}=\varepsilon^{2}-p_{\perp}^{2}-m^{2} .
\end{gathered}
$$

To obtain the symbol $H_{1}(q, \eta)$ of the operator $\hat{\mathcal{O}}_{1}$, we substitute

$$
\frac{\hbar}{i} \frac{\partial}{\partial q} \rightarrow \eta
$$

The result is

$$
\begin{aligned}
& H_{1}(q, \eta)=\frac{1}{2}\left[-\mu^{2} \mathcal{F}(\hat{z})+V(q)\right], \\
& \mathcal{F}(\hat{z})=\hat{z} f(\hat{z}), \quad \hat{z}=\hat{\eta}_{0}^{2}-\hat{\eta}^{2} .
\end{aligned}
$$

For real $\eta$ the variable $\hat{z}$ is also real, as well as the Hamiltonian itself. We also require that for real $\hat{z}$ one has $\mathcal{F}^{\prime}(\hat{z})>0$. For $f(\hat{z})=\exp (g(\hat{z}))$ this condition is satisfied when

$$
1+\hat{z} \frac{d g(\hat{z})}{d \hat{z}}>0
$$

In particular, this is valid for the choice (7).

The Hamilton's equations, (18) and (19) in $(q, \eta)$-phase space reduce to

$$
\dot{q}=\frac{\partial H_{1}}{\partial \eta}=\mu^{2} \mathcal{F}^{\prime} \eta, \quad \dot{\eta}=-\frac{\partial H_{1}}{\partial q}=-\frac{d V}{d q},
$$

while the constraint equation is $H_{1}(q, \eta)=0$. Let us denote by $(q(t), \eta(t))$ a solution of these equations with the initial condition $q(0)=q_{0}$ and $\eta(0)=\eta_{0}$. A solution of the field Eq. (29) in the leading order of the WKB approximation is

$$
\begin{gathered}
\Phi(q)=\frac{1}{\sqrt{J}} \exp \left[\frac{i}{\hbar} \mathcal{S}(q)\right] \Phi_{0}\left(q_{0}\right), \\
\mathcal{S}(q)=\int_{q_{0}}^{q} \eta d q=\int_{0}^{\tau} \eta(\tau) \dot{q}(\tau) d \tau .
\end{gathered}
$$

In the expression for $\mathcal{S}(q)$ the integral is taken along the path $q(\tau)$ connecting the initial point $q_{0}$ with a final point $q$. The function $\Phi_{0}\left(q_{0}\right)=\exp \left[\frac{i}{\hbar} \mathcal{S}\left(q_{0}\right)\right] u_{0}\left(q_{0}\right)$ is the initial value for $\Phi(q)$. The factor $J$ is

$$
J=\left|\frac{d x}{d \tau}\right|=\mu \mathcal{F}^{\prime} \sqrt{\hat{z}_{0}-\hat{z},} \quad \hat{z}_{0}=\hat{\eta}_{0}^{2} .
$$

The second equality in the expression for $J$ is obtained by using the first Hamilton's Eq. (36).

Since the Hamiltonian $H_{1}(q, \eta)$ is invariant under the reflection $\eta \rightarrow-\eta$ one can write another WKB solution by a simple change of the sign in the exponent of (37). Taking a superposition of these two WKB solutions one can write $\Phi(q)$ in the following form

$$
\Phi(q)=\frac{C_{1}}{\sqrt{J}} \exp \left[\frac{i}{\hbar} \mathcal{S}(q)\right]+\frac{C_{2}}{\sqrt{J}} \exp \left[-\frac{i}{\hbar} \mathcal{S}(q)\right] .
$$

For $C_{2}=\bar{C}_{1}$ the WKB solution (40) is real.

\section{BOUND MOTION: ENERGY LEVELS}

\section{A. A general case}

Let us assume that the potential $V(q)$ is nonnegative, and at some point $a$ the following condition is valid,

$$
\hat{\mathcal{F}}\left(\hat{\eta}_{0}\right)=\hat{V}(a) .
$$

Then the constraint equation

$$
\hat{\mathcal{F}}(\hat{z})=\hat{V}(q)
$$

implies that the momentum $\eta$ vanishes at $a$. This means that $a$ is a turning point. If $d V / d q(a)>0$, then the "particle" comes to this point from the left, that is, from the domain where $q<a$ and $\eta>0$. After reaching this turning point $\eta$ changes its sign and the particle moves to the left. If the potential $V(q)$ has another turning point $b$, where $d V / d q(b)<0$, then the particle comes to it from the right with $\eta<0$, changes its direction of motion at $b$ to the opposite, and moves to the right with $\eta>0$.

Let us assume that there exist two turning points $a<b$ such that the motion is restricted by the interval $a \leq q \leq b$. Such a motion is called bound. Let us consider a nonnegative potential $V(q)$ which has its minimum at $q_{0}$ and 


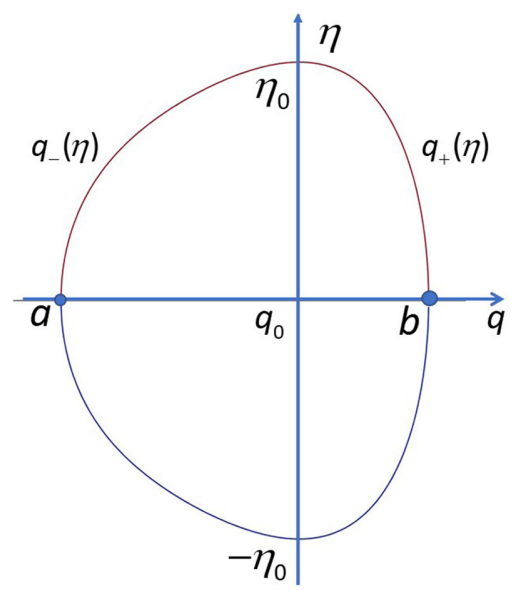

FIG. 2. A phase trajectory.

$V\left(q_{0}\right)=0$. At this point the absolute value of the momentum $|\eta(q)|$ reaches its maximum, which is equal to $\eta_{0}$. Thus, for such a bound motion the phase trajectory lies within a rectangle $[a, b] \times\left[-\eta_{0}, \eta_{0}\right]$. Let us emphasize that the Hamiltonian (33) contains a free parameter $\eta_{0}$, which determines a size and a shape of the phase trajectory. Since the parameter $\hat{z}$ depends on the square of the momentum $\eta$, a phase trajectory on the plane $(q, \eta)$ is symmetric with respect to the reflection $\eta \rightarrow-\eta$. For a given value $\eta_{0}>0$, constraint Eq. (42) defines a closed curve on the $(q, \eta)$ phase plane (see Fig. 2).

The energy levels of the states corresponding to the bound motion are quantized. In the quasiclassical approximation these levels can be found by using standard BohrSommerfeld quantization condition [46]:

$$
J=\oint \eta d q=\pi \hbar(2 n+1)
$$

Here the integral is taken over a complete period. For a given $\eta_{0}>0$, this integral is equal to the surface area inside the phase-trajectory curve corresponding to this parameter. Because of the reflection symmetry $\eta \rightarrow-\eta$, this integral can be written as

$$
J=2 \int_{a}^{b} \eta(q) d q
$$

The integral is taken between the turning points $a$ and $b$.

Sometimes it is convenient to rewrite $J$ in another form. Using constraint Eq. (42) one can express the coordinate $q$ as a function of $\eta$. This function has two branches, which we denote by $q_{ \pm}(\eta)$ (see Fig. 2). Then, integrating by parts (44) and taking into account that $\eta(a)=\eta(b)=0$, one finds

$$
J=2 \int_{0}^{\eta_{0}}\left(q_{+}(\eta)-q_{-}(\eta)\right) d \eta
$$

If the potential $V(x)$ is a symmetric function of $q$, $V(-q)=V(q)$, then one has $q_{0}=0$ and $q_{-}(\eta)=$ $-q_{+}(\eta)$. In this case the quantity $J$ can be written in the form

$$
J=4 \int_{0}^{\eta_{0}} q_{+}(\eta) d \eta
$$

\section{B. Parabolic potential: Local theory}

Let us consider a case when the potential $V(q)$ is parabolic

$$
V(q)=V_{1}^{2} q^{2},
$$

where $V_{1}$ is a positive constant which has the dimension $\left[V_{1}\right]=M / L$. For the local theory the form factor $f(\hat{z})=1$ and the constraint equation takes the form

$$
\eta^{2}+V_{1}^{2} q^{2}=\eta_{0}^{2}
$$

Solving (48) we find

$$
q= \pm V_{1}^{-1} \sqrt{\eta_{0}^{2}-\eta^{2}}
$$

The integral (46) can be easily calculated. We denote the result by $J_{0}$. Then one has

$$
J_{0}=\frac{\pi \eta_{0}^{2}}{V_{1}}
$$

This gives the following expression for the energy levels:

$$
\varepsilon_{n}^{2}=m^{2}+p_{\perp}^{2}+\hbar V_{1}(2 n+1) .
$$

It is easy to see that the number of levels $\Delta n$ within the interval $\Delta\left(\varepsilon^{2}\right)$ is

$$
\Delta n=\frac{1}{2 \hbar V_{1}} \Delta\left(\varepsilon^{2}\right) .
$$

The coefficient $1 /\left(2 \hbar V_{1}\right)$ does not depend on $n$. In this sense, the corresponding distribution of energy levels, $\Delta n / \Delta\left(\varepsilon^{2}\right)$, is equidistant.

\section{Parabolic potential: Nonlocal theory}

\section{Phase trajectories}

In order to discuss the effect of the nonlocality on the distribution of the energy levels for the trapped nonlocal field, we consider the same parabolic potential (47) as in the previous subsection but modify the kinetic part of the effective Hamiltonian. We use dimensionless units defined in Footnote 2 and denote 


$$
\hat{q}=V_{1} q / \mu, \quad \hat{z}_{0}=\hat{\eta}_{0}^{2}=\frac{1}{\mu^{2}}\left(\varepsilon^{2}-m^{2}-p_{\perp}^{2}\right) .
$$

Then the constraint (42) gives

$$
\hat{q}= \pm \hat{\mathcal{F}}(\hat{z})^{1 / 2} .
$$

Let us denote

$$
\hat{\eta}=\hat{\eta}_{0} \sin \phi, \quad \hat{z}=\hat{z}_{0} \cos ^{2} \phi .
$$

Then relations (54) and (55) allow one to write the equation for phase trajectories in the parametric form $(q(\phi), \eta(\phi))$. The phase trajectories for the special choice of the form factor (7) are shown in Fig. 3.

\section{Energy levels}

Using dimensionless variables, the action integral (46), which enters the Bohr-Sommerfeld relation (43) for the energy levels, can be written as follows:

$$
J=\frac{2 \mu^{2}}{V_{1}} \int_{0}^{\hat{z}_{0}} \frac{d \hat{z} \sqrt{z}}{\sqrt{\hat{z}_{0}-\hat{z}}} f^{1 / 2}(\hat{z}) .
$$

We use here the expression for $\hat{\mathcal{F}}$ in terms of the form factor $f, \hat{\mathcal{F}}=\hat{z} f(\hat{z})$. After change of variables, $\hat{z}=\hat{z}_{0} \xi$, relation (56) takes the form

$$
J=\sigma\left(\hat{z}_{0}\right) J_{0}
$$

Here $J_{0}$ is the value of the action integral for the local theory (50) and the factor $\sigma\left(\hat{z}_{0}\right)$ is

$$
\sigma\left(\hat{z}_{0}\right)=\frac{2}{\pi} \int_{0}^{1} \frac{d \xi \sqrt{\xi}}{\sqrt{1-\xi}} f^{1 / 2}\left(\hat{z}_{0} \xi\right) .
$$

For a fixed value of $\eta_{0}$ and $\mu \rightarrow \infty$, the parameter $\hat{z}_{0} \rightarrow 0$. Since $f(0)=1$, the integral (58) simplifies. It can be calculated analytically, and the result is $\sigma(0)=1$. Hence, in this limit $J$ coincides with $J_{0}$. This is not surprising since in the limit $\mu \rightarrow \infty$ the theory becomes local. For a numerical calculation of $\sigma\left(\hat{z}_{0}\right)$, it is convenient to put $\xi=\cos ^{2} \phi$ and rewrite (58) in the form

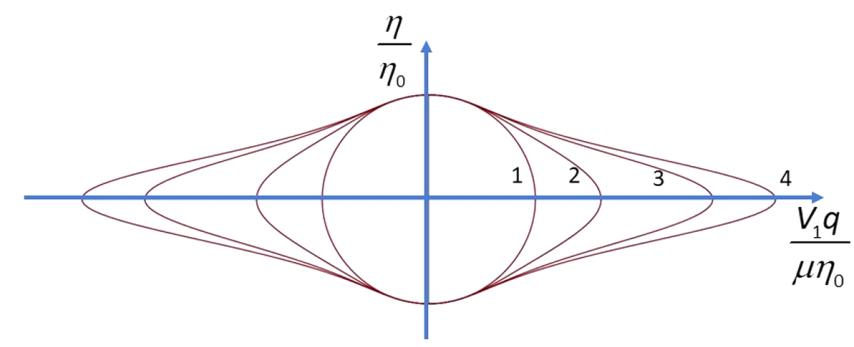

FIG. 3. Phase trajectories for the form factor $f=\exp \left(\hat{z}^{2}\right)$. Different curves correspond to the different values of $\hat{z}_{0}$ parameter: 0.14 for line $1 ; 0.7$ for line $2 ; 1.0$ for line 3 , and 1.1 for line 4 .

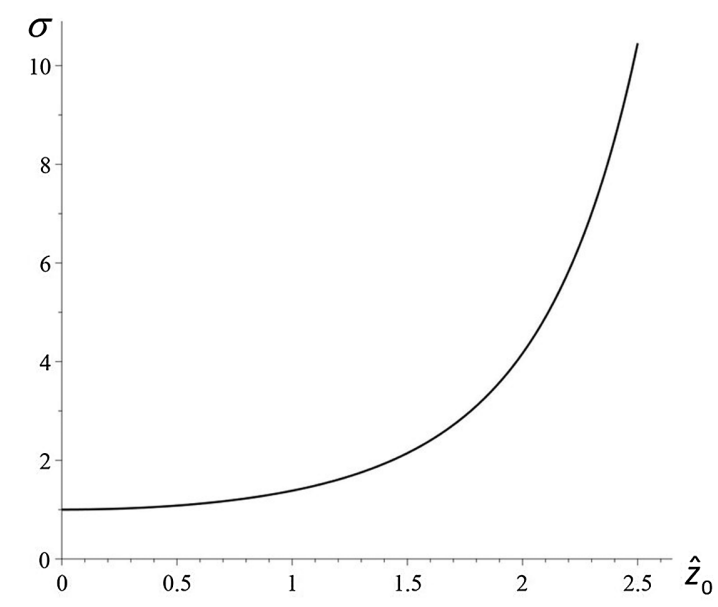

FIG. 4. Function $\sigma\left(\hat{z}_{0}\right)$ for the form factor $f(\hat{z})=\exp \left(\hat{z}^{2}\right)$.

$$
\sigma\left(\hat{z}_{0}\right)=\frac{4}{\pi} \int_{0}^{\pi / 2} d \phi \cos ^{2} \phi f^{1 / 2}\left(\hat{z}_{0} \cos ^{2} \phi\right) .
$$

The function $\sigma\left(\hat{z}_{0}\right)$ for the choice of the form factor $f(\hat{z})=\exp \left(\hat{z}^{2}\right)$ is shown at Fig. 4. For this form factor it is possible to show that for large $\hat{z}_{0}$ the function $\sigma\left(\hat{z}_{0}\right)$ has the following asymptotic form:

$$
\sigma\left(\hat{z}_{0}\right) \sim \frac{1.13}{\hat{z}_{0}} \exp \left(\hat{z}_{0}^{2} / 2\right) .
$$

Figure 5, which plots the ratio $\sigma\left(\hat{z}_{0}\right) / \sigma_{0}\left(\hat{z}_{0}\right)$, demonstrates this property.

The parameter $\hat{z}_{0}$, which enters $J$, depends on the energy $\varepsilon$. The condition of quantization

$$
J=\pi \hbar(2 n+1)
$$

allows one to find the corresponding quasiclassical energy levels $\varepsilon_{n}$. Since for small $\hat{z}_{0}$ the coefficient $\sigma\left(\hat{z}_{0}\right)$ is close to 1 , the number of the energy levels $n$ in the interval $\Delta \varepsilon^{2}$ has the form (52). However, for large $n$ this distribution of the number of levels is quite different. For the form factor $f(\hat{z})=\exp \left(\hat{z}^{2}\right)$, one can find it by using the asymptotic form of the coefficient $\sigma\left(\hat{z}_{0}\right)$,

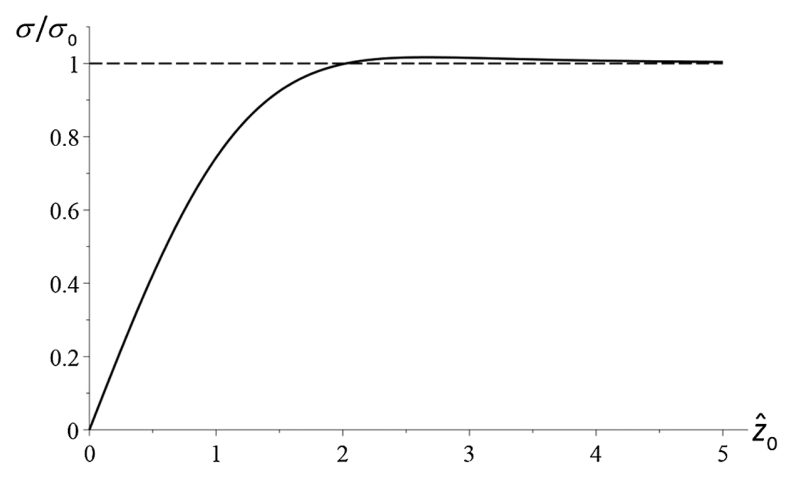

FIG. 5. Ratio $\sigma / \sigma_{0}$ as a function of $\hat{z}_{0}$. 


$$
J=1.13 \frac{\pi \mu^{2}}{V_{1}} \exp \left(\hat{z}_{0}^{2} / 2\right) .
$$

The condition of quantization (61) gives

$$
\exp \left(\hat{z}_{0}^{2} / 2\right)=0.88 \frac{\hbar V_{1}}{\mu^{2}}(2 n+1) .
$$

For $n \gg 1$ one gets

$$
\hat{z}_{0}^{2} \approx 2 \ln n \text {. }
$$

Since $\hat{z}_{0}=\eta_{0}^{2} / \mu^{2}$, one finds

$$
\varepsilon^{2} \approx m^{2}+p_{\perp}^{2}+\sqrt{2} \mu^{2} \sqrt{\ln n} .
$$

This relation implies

$$
\Delta n \approx \frac{\sqrt{2}}{\mu^{2}} n \sqrt{\ln n} \Delta\left(\varepsilon^{2}\right) .
$$

The obtained result means that the equidistance of the energy level distribution (52) of the local theory is broken by nonlocality. In the limit of large $n$ and given interval $\Delta\left(\varepsilon^{2}\right)$ the number of the corresponding levels $\Delta n$ grows with $n$ as $n \sqrt{\ln n}$.

\section{BARRIER PENETRATION}

\section{A. WKB approximation for under-barrier "motion"}

Let us consider a potential which has the form shown in Fig. 6 and assume that the effective energy of the particle is such that its trajectory has a turning point at $q=a$. At this point one has

$$
\mathcal{F}\left(\hat{z}_{0}\right)=\hat{V}(a), \quad \hat{z}_{0}=\hat{\eta}_{0}^{2} .
$$

Let us write the constraint Eq. (42) in the form

$$
\mathcal{F}\left(\hat{z}_{0}\right)-\mathcal{F}(\hat{z})=\hat{V}(a)-\hat{V}(q) .
$$

In the domain to the left from the turning point $a$, one has $\hat{V}(q)<\hat{V}(a)$. We assume that for real $\hat{z}$ the function $\mathcal{F}(\hat{z})$

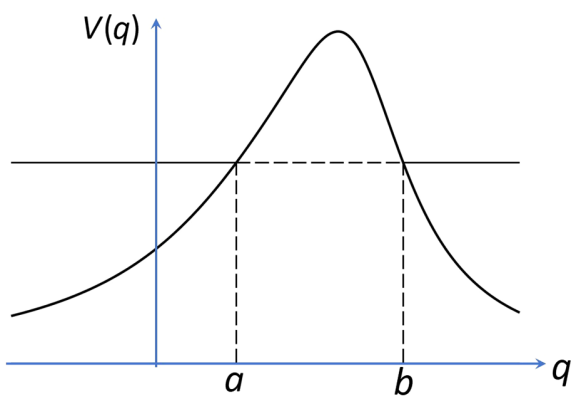

FIG. 6. Potential $V(q)$. is monotonically growing. Then one has $\hat{z}<\hat{z}_{0}$. In the region $q>a$, where $\hat{V}(q)>\hat{V}(a)$, one should have $\hat{z}>\hat{z}_{0}$. This condition implies that $\eta^{2}<0$, which is impossible for a real value of $\eta$.

It is well known that in the local theory a quasiclassical solution for a wave function in the classically forbidden domain can be obtained by considering complex trajectories. Namely, one should change $\eta \rightarrow i \eta$. The same trick does work for the nonlocal theories considered in this paper. After the change $\hat{z} \rightarrow \hat{\eta}_{0}^{2}+\hat{\eta}^{2}$, the value of $\hat{z}$ can be larger than $\hat{z}_{0}$ for real $\hat{\eta}$, and Eq. (68) has a solution in the region, where $q>a$, that is in the classically forbidden domain.

A quasiclassical solution in the underbarrier domain can be obtained by the change $\eta \rightarrow i \eta$ in (40), and it is of the form

$$
\begin{gathered}
\Phi(q)=\frac{C_{-}}{\sqrt{J}} \exp \left[-\frac{1}{\hbar} \mathcal{S}(q)\right]+\frac{C_{+}}{\sqrt{J}} \exp \left[\frac{1}{\hbar} \mathcal{S}(q)\right] \\
\mathcal{S}(q)=\int_{q_{0}}^{q} \eta d q=\int_{0}^{\tau} \eta(\tau) \dot{q}(\tau) d \tau .
\end{gathered}
$$

Here,

$$
J=\mu \mathcal{F}^{\prime} \sqrt{\hat{z}-\hat{z}_{0}}, \quad \hat{z}_{0}=\hat{\eta}_{0}^{2} .
$$

For real coefficients $C_{ \pm}$the WKB solution (69) is real.

\section{B. Nonlocal field in a linear potential}

For a linear potential

$$
V(q)=U_{0}+U_{1}(q-a),
$$

one-dimensional nonlocal field Eq. (29) can be solved exactly. For this purpose we denote $q=a+x$ and write $\Phi(x)$ in the form

$$
\Phi(x)=\int_{-\infty}^{\infty} d \eta e^{i \eta x / \hbar} \tilde{\Phi}(\eta) .
$$

Using the inverse Fourier transform, one gets

$$
\tilde{\Phi}(\eta)=\frac{1}{2 \pi \hbar} \int_{-\infty}^{\infty} d x e^{-i \eta x / \hbar} \Phi(x) .
$$

The field Eq. (29) implies the following differential equation for $\tilde{\Phi}(\eta)$ :

$$
\left[U_{1} \frac{\hbar}{i} \frac{\partial}{\partial \eta}+U_{0}+\mu^{2} \mathcal{F}(\hat{z})\right] \tilde{\Phi}(\eta)=0 .
$$

A solution of this equation is 


$$
\begin{aligned}
& \tilde{\Phi}(\eta)=C \exp \left[-\frac{i}{\hbar U_{1}} \mathcal{S}(\eta)\right] \\
& \mathcal{S}(\eta)=U_{0} \eta+\mu^{2} \int_{0}^{\eta} d \eta \mathcal{F}(\hat{z}) .
\end{aligned}
$$

Substitution of this expression into (73) gives

$$
\Phi(x)=C \int_{-\infty}^{\infty} d \eta \exp \left[\frac{i}{\hbar}\left(\eta x-\frac{1}{U_{1}} \mathcal{S}(\eta)\right)\right] .
$$

This is a desired solution of the nonlocal field Eq. (29).

\section{Connection of WKB solutions at a turning point}

Consider a potential of the form shown in Fig. 6 and denoted by $a$, a coordinate of a left turning point. One has $V_{, q}(a)>0$. To the left of $a$ and at some distance from it one can use WKB solution (40). For the real field $\Phi(q)$, we denote

$$
C_{1}=\frac{1}{2} C \exp (-i \psi), \quad C_{2}=\frac{1}{2} C \exp (i \psi),
$$

where $C$ and $\psi$ are real and write the WKB solution (40) in the form

$$
\begin{gathered}
\Phi(q)=\frac{C}{\sqrt{J}} \cos (\mathcal{S} / \hbar-\psi) \\
\mathcal{S}=\int_{a}^{q} \eta(q) d q, \quad J=\mu \mathcal{F}^{\prime} \sqrt{\hat{z}_{0}-\hat{z}}
\end{gathered}
$$

To the right of the turning point $a$ and at some distance from it we use a decreasing in the underbarrier domain WKB solution

$$
\begin{gathered}
\Phi(q)=\frac{C_{-}}{\sqrt{J}} \exp (-\mathcal{S} / \hbar) \\
\mathcal{S}=\int_{a}^{q} \eta(q) d q, \quad J=\mu \mathcal{F}^{\prime} \sqrt{\hat{z}-\hat{z}_{0}}
\end{gathered}
$$

At the turning point the WKB approximation is not valid. In order to establish a relation between the (80) and (82) solutions, one can use the following trick [48] which can be easily adapted to our problem. For this purpose we consider formally $\Phi(q)$ as a function of a complex variable $q$ and find it along a path on the complex plane of $q$ connecting positive and negative values of $q-a$. We choose this path to be a half of the circle of constant radius $\rho$ with the center at $q=a$ and take $\rho$ so that the conditions of the validity of the WKB approximation are satisfied along the path. Let us denote

$$
q-a=\rho e^{ \pm \phi}
$$

We choose a sign + for a semicircle in the upper half of the complex $q$-plane and - for a semicircle in the lower half of the complex $q$-plane. The angle $\phi$ changes from 0 to $\pi$. At the turning point, $\hat{z}=\hat{z}_{0}=\hat{\eta}_{0}^{2}$ and $q=a$. We assume that $\rho$ is small so that $\left|\hat{z}-\hat{z}_{0}\right|$ and $|q-a|$ are also small and use the following linearized form of the constraint equation:

$$
\mathcal{F}_{0}^{\prime}\left(\hat{z}-\hat{z}_{0}\right)=\hat{V}_{, q}(a)(q-a), \quad \mathcal{F}_{0}^{\prime}=\left.(d \mathcal{F} / d \hat{z})\right|_{\hat{z}_{0}} .
$$

Let us denote $B=\sqrt{\hat{V}_{, q}(a) / \mathcal{F}_{0}^{\prime}}$. One also has $\hat{z}-\hat{z}_{0}=\hat{\eta}^{2}$. Then (84) and (85) imply

$$
\eta=\mu B \rho^{1 / 2} e^{ \pm i \phi / 2} .
$$

For the analytically continued WKB solution (82), we get

$$
\begin{aligned}
\mathcal{S}^{ \pm} & =\frac{2}{3} \mu B \rho^{3 / 2} \exp ( \pm 3 i \phi / 2), \\
J^{ \pm} & =\mu B \mathcal{F}_{0}^{\prime} \rho^{1 / 2} \exp ( \pm i \phi / 2) .
\end{aligned}
$$

Here \pm stands for the quantities calculated along a semicircle in the upper (+) and lower (-) half of the $q$-plane. Using these relations at $\phi=\pi$ where $a-q=\rho$, one obtains

$$
C=\frac{1}{2} C_{-}, \quad \psi=-\pi / 4 .
$$

This establishes a relation between the WKB solution (82) in the domain to the right of the turning point and the WKB solution (80) to the left of it. This gives a required connection formula. It should be emphasized that for the operator $F(\square)$ with the properties specified in Sec. II, this connection relation is the same as for a standard Schrödinger equation.

We assume now that the potential has two turning points $a<b$ and the domain between these points is forbidden for the classical motion. We suppose that in the region $q<a$ the field $\Phi(q)$ has the form (80) and its amplitude is $C$. Then in the region $q>b$ it has the same form with a different amplitude $\tilde{C}$. Similarly to the local case, the ratio $|\tilde{C} / C|^{2}$ can be found by using the connection formula (89) twice [48]

$$
\begin{gathered}
R=\left(\frac{\tilde{C}}{C}\right)^{2}=\exp \left(-\frac{2}{\hbar} P\right), \\
P=\mu \int_{a}^{b} \sqrt{\hat{z}-\hat{z}_{0}} d q .
\end{gathered}
$$

Here, $\hat{z}_{0}=\hat{\eta}_{0}^{2}$ and $\hat{z}=\hat{z}_{0}^{2}+\hat{\eta}^{2}$. In the expression for $P$ the quantities $\hat{z}$ and $q$ satisfy the constraint (42). The quantity $R$ gives a probability of the barrier penetration. It should be 
emphasized that these relations are obtained in the WKB approximation, which is valid when $R \ll 1$.

\section{Barrier penetration}

\section{Inverse parabolic potential: Local theory}

For the local theory the effective Hamiltonian is

$$
H(q, \eta)=\frac{1}{2}\left[-\left(\eta_{0}^{2}-\eta^{2}\right)+V(q)\right],
$$

where $\eta_{0}^{2}=\varepsilon^{2}-p_{\perp}^{2}-m^{2}$. Let the potential $V(q)$ be of the form

$$
V(q)= \begin{cases}V_{0}-V_{1}^{2} q^{2}, & \text { if }|q| \leq q_{*} \\ 0, & \text { if }|q|>q_{*}\end{cases}
$$

where $V_{0}>0$ and $q_{*}=\sqrt{V_{0}} / V_{1}$. This potential is shown in Fig. 7. It is called an inverse parabolic potential.

We assume that $\eta_{0}^{2}<V_{0}$ so that there exit two turning points at $q= \pm a$, where

$$
a=\frac{\sqrt{V_{0}-\eta_{0}^{2}}}{V_{1}} .
$$

For the motion in the underbarrier domain, we put $\eta=i \tilde{\eta}$ where

$$
\tilde{\eta}= \pm \sqrt{V-\eta_{0}^{2}}= \pm V_{1} \sqrt{a^{2}-q^{2}}
$$

We denote by $P_{0}$ the following integral:

$$
\begin{aligned}
P_{0} & =\int_{-a}^{a}|\tilde{\eta}| d q \\
& =V_{1} \int_{-a}^{a} d q \sqrt{a^{2}-q^{2}}=\frac{1}{2} \pi a^{2} V_{1} .
\end{aligned}
$$

Using relation (94) we can also write $P_{0}$ in the form

$$
P_{0}=\frac{\pi\left(V_{0}-\eta_{0}^{2}\right)}{2 V_{1}}
$$

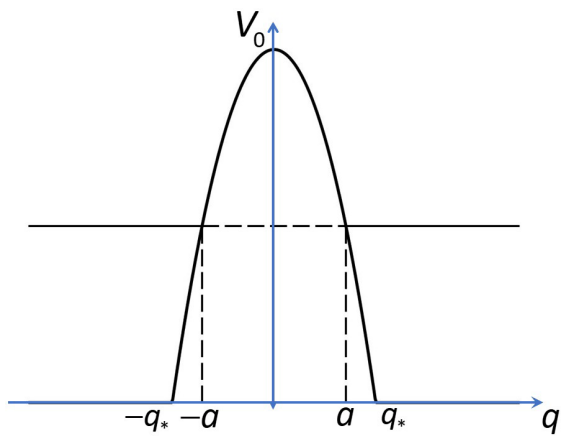

FIG. 7. The inverse parabolic potential.
Let us emphasize that $P_{0}$ depends only on the difference $V_{0}-\eta_{0}^{2}$, but it does not depend on the value of $\eta_{0}$ itself. In other words, the penetration probability is sensitive only to the fact of how close the effective energy $\eta_{0}^{2}$ is to the top of the potential $V_{0}$. Let us also remind that the adopted WKB approximation is valid only when the dimensionless quantity $P_{0}$ is large, that is, when the probability of the penetration $R_{0}=\exp \left(-2 P_{0} / \hbar\right)$ is small (see e.g., [48]).

\section{Inverse parabolic potential: Nonlocal theory}

Let us assume again that the potential $V(q)$ has the form (93). We denote by $\hat{z}_{0}$ and $\hat{z}_{1}$ solutions of the following equations:

$$
\mathcal{F}\left(\hat{z}_{0}\right)=\hat{V}_{0}-\hat{V}_{1}^{2} a^{2}, \quad \mathcal{F}\left(\hat{z}_{1}\right)=\hat{V}_{0} .
$$

Then the WKB probability of the barrier penetration is

$$
\begin{gathered}
R=\exp \left(-\frac{2}{\hbar} P\right) \\
P=\frac{\mu}{\hat{V}_{1}} \int_{\hat{z}_{0}}^{\hat{z}_{1}} \frac{d \hat{z}}{\sqrt{\hat{z}-\hat{z}_{0}}} \sqrt{\mathcal{F}\left(\hat{z}_{1}\right)-\mathcal{F}(\hat{z})} .
\end{gathered}
$$

Let us calculate the value of $P$ for the case when $\mathcal{F}$ is of the form (7). When both parameters $V_{0}$ and $\eta_{0}^{2}$ are much smaller than $\mu^{2}$, the function $X(\hat{z}) \approx \hat{z}$ and the integral in (100) can be easily taken with the following result:

$$
P=\frac{\pi \mu\left(\hat{z}_{1}-\hat{z}_{0}\right)}{2 \hat{V}_{1}}=\frac{\pi\left(V_{0}-\eta_{0}^{2}\right)}{2 V_{1}} .
$$

In this regime the effects of the nonlocality are not important, and we reproduce the expression for $P_{0}$ obtained in the local case [see Eq. (97)].

The effects of the nonlocality play an important role in the different regime when $\hat{V}_{0} \gg 1$ and $\hat{\eta}_{0}^{2} \gg 1$. To demonstrate this we consider again the theory with the form factor $f(\hat{z})=\exp \left(\hat{z}^{2}\right)$. In order to compare the expression for $P$ with $P_{0}$ we fix as earlier the value of the difference $V_{0}-\eta_{0}^{2}$, but now we assume that $\hat{V}_{0} \gg 1$. Then one has $\hat{z}_{1}-\hat{z}_{0} \ll 1$. In Appendix B it is shown that one can write the following approximate expression for $P$ in this case:

$$
P=\kappa\left(\hat{V}_{0}\right) P_{0}, \quad \kappa\left(\hat{V}_{0}\right)=\frac{1}{\sqrt{X_{1}^{\prime}}} .
$$

Here, $X_{1}^{\prime}=d X /\left.d x\right|_{x=\hat{z}_{1}}$ and $X\left(\hat{z}_{1}\right)=\hat{V}_{0}$. Using (B2) one can write

$$
\begin{gathered}
X_{1}^{\prime}\left(\hat{z}_{1}\right)=\left(1+2 \hat{z}_{1}^{2}\right) \exp \left(\hat{z}_{1}^{2}\right), \\
\hat{V}_{0}=\hat{z}_{1}^{2} \exp \left(\hat{z}_{1}^{2}\right)
\end{gathered}
$$




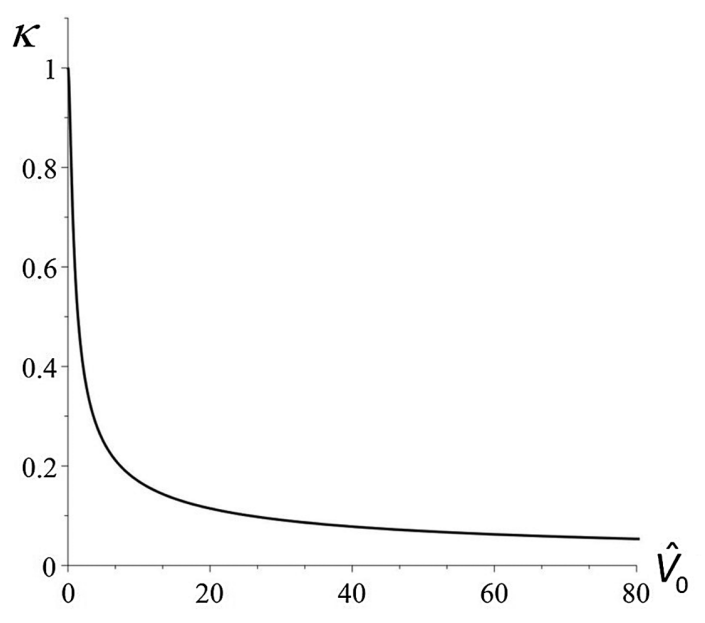

FIG. 8. Parameter $\kappa$ as a function of $\hat{V}_{0}$.

These relations give a parametric representation of $X_{1}^{\prime}\left(\hat{z}_{1}\right)$ as a function of $\hat{V}_{0}$. The plot of the function $\kappa\left(\hat{V}_{0}\right)$ is shown in Fig. 8. It shows that this function is equal to 1 at $\hat{V}_{0}=0$, and it is less than 1 and monotonically decreases for positive $\hat{V}_{0}$.

Since $R=\exp (-2 P / \hbar)$, the relation (102) can be rewritten as follows:

$$
R=\left(R_{0}\right)^{\kappa\left(\hat{V}_{0}\right)} .
$$

Since $R_{0}<1$, the value of $R$ is always greater that $R_{0}$. This means that in the nonlocal theory the probability of the barrier penetration is larger than in the local case. In other words nonlocality makes the barrier more transparent in the high-energy regime.

\section{DISCUSSION}

Let us discuss the obtained results. In this paper we study quasiclassical solutions of the nonlocal massive scalar field equations. The kinetic part of the action for such a theory contains a function of the $\square$-operator, which is chosen so that the theory does not contain new unphysical degrees of freedom. In Sec. II we formulated conditions imposed on the form factor of such a theory. We also described a special model which is used later in the paper for the illustration. In Sec. III we described an ansatz used in the WKB approximation for a solution of the nonlocal field equation. In the leading order of the $1 / \hbar$-expansion one obtains the equation for the eikonal function $S(x)$, which is of the form $H(x, \nabla S)=0$. This is a first-order partial differential equation, which has the form of the Hamilton-Jacobi equation for the Hamiltonian $H(x, p)$, obtained by substitution $\nabla S=p$. To calculate the eikonal function $S(x)$ it is sufficient to construct the Lagrangian submanifold in the phase space $(x, p)$ by using the initial conditions for $S$. Such a Lagrangian submanifold is formed by phase trajectories which are solutions of the Hamiltonian equations of motion. Since the system is conservative, this submanifold belongs to the subspace $H(x, p)=0$. Let us emphasize that this procedure is very similar to the standard one adopted for the quantum mechanics and local field theory. When the field operator is of the second order the corresponding effective Hamiltonian $H(x, p)$ is a quadratic polynomial in momentum $p$. For the nonlocal theory $H(x, p)$ is a nonpolynomial function of $p$, and this is a main difference with the local case. As a result the construction of the WKB solutions becomes technically more complicated.

WKB solutions presented in Sec. III are valid for a wide class of nonlocal scalar field theories with an arbitrary external potential $V(x)$ in a flat spacetime with any number of spatial dimensions. These relations are greatly simplified for the case when the potential $V$ depends only on one spatial Cartesian coordinate. For this case the phase space is two dimensional and the Hamilton's equations are completely integrable. The corresponding WKB solutions are described in Sec. IV. In Sec. V we used the WKB approximation for the calculation of the energy levels of the nonlocal field trapped by a one-dimensional potential. To illustrate general formulas we considered the case when the form factor of the nonlocality is of the special form (7), and the potential is parabolic. The energy levels $\varepsilon_{n}$ of the field depend on a discrete integer parameter $n$. For the local case the number $\Delta n$ of the levels in the interval $\Delta\left(\varepsilon^{2}\right)$ is $\Delta n=\Delta\left(\varepsilon^{2}\right) /\left(2 \hbar V_{1}\right)$, and it does not depend on the value of $n$. In this sense the levels are equidistant. In the nonlocal case for large $n$ this dependence is different

$$
\Delta n \approx \frac{\sqrt{2}}{\mu^{2}} n \sqrt{\ln n} \Delta\left(\varepsilon^{2}\right) .
$$

This means that for a fixed value of $\frac{\hbar V_{1}}{\mu^{2}}$ and very high energy there are much more energy levels in a given interval $\Delta\left(\varepsilon^{2}\right)$ than in the local case.

Finally, we discussed the under-barrier motion and obtained an expression for the probability of the barrier penetration. This probability has the form $\sim \exp (-2 P / \hbar)$. We calculated the factor $P$ for the special form of the nonlocality (7) in the presence of the inverse parabolic potential. For the local theory this factor is $P_{0}=$ $\pi\left(V_{0}-\eta_{0}^{2}\right) /\left(2 V_{1}\right)$. In other words, it depends on how close is the energy of the state $\eta_{0}^{2}$ to the top of the potential $V_{0}$, but it does not depend on the height $V_{0}$ of the potential itself. The situation in the nonlocal case is quite different. Namely, one has $P=\kappa P_{0}$, where the factor $\kappa$ depends on the height $V_{0}$ of the potential. This factor is always less than 1 , and it decreases when $V_{0}$ grows. This means that the nonlocality "helps" the field to penetrate the potential barrier.

The examples considered in this paper are rather simple. It would be interesting to apply the developed in the paper 
method for study of the effects of the nonlocality in the early cosmology and in the black hole's interior.

\section{ACKNOWLEDGMENTS}

The author thanks the Natural Sciences and Engineering Research Council of Canada and the Killam Trust for their financial support.

\section{APPENDIX A: WKB APPROXIMATION}

We collected here useful information concerning construction of solutions of the nonlocal field equations in the WKB approximation. Further details and required mathematical foundations can be found in the book [46].

Let $\left(x^{1}, \ldots, x^{N}\right)$ be coordinates of a point $x$ in $R^{N}$. We denote by $\alpha$ a set of $N$ non-negative integer numbers $\alpha=\left(\alpha_{1}, \ldots, \alpha_{N}\right)$. We consider the following operator in this space:

$$
\hat{\mathcal{O}}=\sum_{\alpha} a_{\alpha}\left(\frac{\hbar}{i} \frac{\partial}{\partial x^{1}}\right)^{\alpha_{1}} \ldots\left(\frac{\hbar}{i} \frac{\partial}{\partial x^{N}}\right)^{\alpha_{N}}
$$

The sum is taken over all different combinations of $\alpha$. We do not assume that the number of terms in the sum is finite, so that this expression can be used as a formal series in derivatives representation of nonlocal operators.

Let us make a substitution

$$
\frac{\hbar}{i} \frac{\partial}{\partial x^{\mu}} \rightarrow p_{\mu}
$$

in the expression (A1). Then we obtain a function of $2 N$ variables which we denote by $H=H(x, p)$,

$$
H=H(x, p)=\sum_{\alpha} a_{\alpha} p_{1}^{\alpha_{1}} \ldots p_{N}^{\alpha_{N}} .
$$

Here, $p=\left(p_{1}, \ldots, p_{N}\right)$. This object is called a symbol of the operator $\hat{\mathcal{O}}$.

We are looking for a solution $\varphi$ of the equation

$$
\hat{\mathcal{O}} \varphi=0
$$

in the form of the following formal series:

$$
\varphi(x)=\exp \left(\frac{i S(x)}{\hbar}\right) \sum_{j=0}^{\infty}\left(\frac{\hbar}{i}\right)^{j} u_{j}(x) .
$$

Usually it is sufficient to consider only the first few terms in the series. This gives what is known as the WKB approximation for the solution $\varphi$. To obtain the functions $S(x)$ and $u_{j}(x)$ that enter (A5) the following procedure is used (see e.g., $[45,46]$ and references therein).

If one substitutes expression (A5) into the Eq. (A4), then one finds that in the leading order $j=0$ the following condition is to be satisfied:

$$
H\left(x, \frac{\partial S}{\partial x}\right) u_{0}=0
$$

where

$$
\frac{\partial S}{\partial x}=\left(\frac{\partial S}{\partial x^{1}} \ldots, \frac{\partial S}{\partial x^{N}}\right)
$$

For a nonvanishing function $u_{0}(x)$, the following HamiltonJacobi equation is valid:

$$
H\left(x, \frac{\partial S}{\partial x}\right)=0
$$

Let us consider a $(2 N)$-dimension space $\Gamma^{2 N}$ with coordinates $(x, p)=\left(x^{1}, \ldots, x^{N}, p_{1}, \ldots, P_{N}\right)$ and denote by $\Omega$ a symplectic two form in it

$$
\Omega=\sum_{\mu=1}^{N} d p_{\mu} \wedge d x^{\mu}
$$

An $N$-dimensional surface $\Lambda^{N}$ in $\Gamma^{2 N}$ is called a Lagrangian submanifold if the $\Omega$ vanishes on $\Lambda^{N}$. If a Lagrangian submanifold can be uniquely projected onto the $x$-space, then there exists such a function $S(x)$ that $p_{\mu}=\partial S / \partial_{\mu}$ on $\Lambda^{N}$.

The Hamiltonian $H(x, p)$ given by the equation (A3) implies the following Hamilton's equations

$$
\frac{d x^{\mu}}{d \tau}=\frac{\partial H(x, p)}{\partial p_{\mu}}, \quad \frac{d p_{\mu}}{d \tau}=-\frac{\partial H(x, p)}{\partial x^{\mu}} .
$$

These equations determine the Hamiltonian flow in the phase space $\Gamma^{2 N}$ preserving the symplectic form $\Omega$, and the Hamiltonian itself is an integral of motion.

Let $\Sigma^{N-1}$ be a $(N-1)$-dimensional surface in the coordinate space. Its embedding is defined by the equations

$$
x^{\mu}=f^{\mu}\left(y^{i}\right), \quad i=1, \ldots, N-1 .
$$

One can consider $y^{i}$ as coordinates on $\Sigma^{N-1}$. We associate with $\Sigma^{N-1}$ a $(2 N-2)$-dimensional phase space $\Gamma^{2 N-2}$ and denote its canonical coordinates by $(y, v)=$ $\left(y^{1}, \ldots, y^{N-1}, v_{1}, \ldots, v_{N-1}\right)$. The symplectic 2 -form $\omega$ in $\Gamma^{2 N-2}$ is

$$
\omega=\sum_{i=1}^{N-1} d v_{i} \wedge d y^{i}
$$

Denote by $S^{0}=S^{0}(y)$ a function on $\Sigma^{N-1}$ and $\nabla S^{0}=\left(S_{, y^{1}}^{0}, \ldots, S_{, y^{N-1}}^{0}\right)$, then a $(N-1)$-dimensional surface $(y, \nabla S)$ of $\Gamma^{2 N-2}$ is a Lagrangian submanifold $\Lambda^{N-1}$, so that $\left.\omega\right|_{\Lambda^{N-1}}=0$. 
Let us define a covector $p(y)=p_{\mu}(y)$ on $\Sigma^{N-1}$ by two conditions

$p_{\mu}(y) d x^{\mu}=p_{\mu}(y) \frac{\partial f^{\mu}}{\partial y^{i}}=0, \quad H(x(y), p(y))=0$.

We assume that this system of $N$ equations has a solution which determines $\left.p_{\mu}(y)\right|_{\Sigma^{N-1}}$.

Let us consider the following initial conditions for the Hamilton's Eqs. (A10)

$$
\left.x^{\mu}\right|_{\tau=0}=f^{\mu}\left(y^{i}\right),\left.\quad p_{\mu}\right|_{\tau=0}=p_{\mu}(y) .
$$

A family of solutions of the Hamilton's Eqs. (A10) with these initial conditions, $(x(\tau, y), p(\tau, y))$, forms a $N$-dimensional surface in $\Gamma^{2 N}$, which we denote by $\mathcal{B}$. It is possible to show that $\mathcal{B}$ is a Lagrangian submanifold in $\Gamma^{2 N}$ and that $H(x, p)=0$ on it. This implies that there exists such a function $S(x)$ that

$$
\left.p_{\mu}\right|_{\mathcal{B}}=\frac{\partial S}{\partial x^{\mu}}
$$

This function is a solution of the Hamilton-Jacobi Eq. (A8) satisfying the initial condition $\left.S(x)\right|_{\Sigma^{N-1}}=S^{0}(y)$.

This function $S(x)$ can be found as follows. Denote a 1 -form $\theta=\sum_{\mu} p_{\mu} d x^{\mu}$, then $\Omega=d \theta$. Since the symplectic form $\Omega$ vanishes on $\mathcal{B}$, the integral

$$
\int p_{\mu} d x^{\mu}
$$

calculated along a path on $\mathcal{B}$ between its two points does not depend on the choice of the path. The required solution $S(x)$ of the Hamilton-Jacobi equation satisfying the initial condition $\left.S\right|_{\Sigma^{N-1}}=S(y)$ can be written in the form

$$
S(x)=S^{0}(y)+\int_{0}^{\tau} p_{\mu} d x^{\mu} .
$$

Here $x^{\mu}=x^{\mu}(\tau, y)$ and $p_{\mu}=p_{\mu}(\tau, y)$.

Let us note that the map $x^{\mu}=x^{\mu}(\tau, y)$ may be considered as a transformation between the coordinates $(\tau, y)$ and $x^{\mu}$. Let us denote by $J$ a Jacobian of this transformation

$$
J(\tau, y)=\left|\operatorname{det} \frac{\partial x(\tau, y)}{\partial(\tau, y)}\right| .
$$

Then the leading term of the asymptotic solution (A5) is [46]

$$
\begin{aligned}
\varphi(x)= & u_{0}(y) \sqrt{\frac{J(0, y)}{J(\tau, y)}} \exp \left[\frac{i}{\hbar}\left(S^{0}(y)+\int_{0}^{\tau} p_{\mu} d x^{\mu}\right)\right. \\
& \left.-\frac{1}{2} \int_{0}^{\tau} \sum_{\mu=1}^{N} \frac{\partial^{2} H(x, p)}{\partial x^{\mu} \partial p_{\mu}} d \tau\right] .
\end{aligned}
$$

For $\tau=0$ the right-hand side of this expression reduces to $u_{0}(y) \exp \left[\frac{i}{\hbar} S^{0}(y)\right]$, and it should be found from the initial condition for the function $\varphi(x)$ at $\Sigma$.

\section{APPENDIX B: FUNCTION $X(x)=x \exp \left(x^{2}\right)$ AND ITS PROPERTIES}

In this Appendix we discuss some properties of the function

$$
X(x)=x \exp \left(x^{2}\right)
$$

The derivatives of this function are

$$
\begin{aligned}
X^{\prime} & =\left(1+2 x^{2}\right) \exp \left(x^{2}\right) \\
X^{\prime \prime} & =6 x\left(1+\frac{2}{3} x^{2}\right) \exp \left(x^{2}\right), \\
\alpha & =\frac{1}{2} \frac{X^{\prime \prime}}{X^{\prime}}=x\left(1+\frac{2}{2 x^{2}+1}\right) .
\end{aligned}
$$

We denote by $Y$ an inverse function of $X$ such that the following relations are valid:

$$
y=X(x), \quad x=Y(y) .
$$

The inverse function can be written as follows:

$$
Y(y)=y \exp \left(-\frac{1}{2} W\left(2 y^{2}\right) .\right) .
$$

Here, $W$ is a Lambert $W$ function defined by the relation

$$
W(z) \exp (W(z))=z .
$$

For a real positive argument $W$ is a real single-values function.

Consider the following integral:

$$
I=\int_{x_{0}}^{x_{1}} \frac{d x}{\sqrt{x-x_{0}}} \sqrt{X\left(x_{1}\right)-X(x)} .
$$

Let us assume that $x_{1}-x_{0}$ is small. To obtain an approximate value of the integral $I$, in this case, we use the Taylor expansion

$$
X(x)=X_{1}-X_{1}^{\prime}\left(x_{1}-x\right)+\frac{1}{2} X_{1}^{\prime \prime}\left(x_{1}-x\right)^{2}+\ldots
$$


Here, $X_{1}=X\left(x_{1}\right), X_{1}^{\prime}=X^{\prime}\left(x_{1}\right), X_{1}^{\prime \prime}=X^{\prime \prime}\left(x_{1}\right)$. Skipping the higher order terms denoted in (B7) by dots, one has

$$
I=\sqrt{X_{1}^{\prime}} \int_{x_{0}}^{x_{1}} \frac{d x}{\sqrt{x-x_{0}}} \sqrt{\left(x_{1}-x\right)-\alpha\left(x_{1}-x\right)^{2}},
$$

where $\alpha=\frac{1}{2}\left(X_{1}^{\prime \prime} / X_{1}^{\prime}\right)$. This integral can be calculated exactly. Let us denote $\beta=x_{1}-x_{0}$, then for $\alpha \beta<1$ the answer is

$$
I=\frac{2 \sqrt{X_{1}^{\prime}}}{3 \alpha}[(2 \alpha \beta-1) E(\sqrt{\alpha \beta})-(\alpha \beta-1) K(\sqrt{\alpha \beta})] .
$$

Here, $E$ and $K$ are complete elliptic integrals. For the small value of $\alpha \beta$ one has

$$
I=\frac{\pi \beta}{2} \sqrt{X_{1}^{\prime}}\left(1-\frac{3}{8} \alpha \beta\right)
$$

For $\alpha \beta \ll 1$ one can neglect the second term. Keeping the leading term one gets

$$
I=\frac{\pi \beta}{2} \sqrt{X_{1}^{\prime}}=\frac{\pi}{2} \frac{X_{1}-X_{0}}{\sqrt{X_{1}^{\prime}}} .
$$

For small $x_{1}$ one has $X_{1}^{\prime} \approx 1$ and

$$
I \approx \frac{\pi}{2}\left(X_{1}-X_{0}\right)
$$

[1] G. Wataghin, Z. Phys. 88, 92 (1934).

[2] H. Yukawa, Phys. Rev. 77, 219 (1950).

[3] H. Yukawa, Phys. Rev. 80, 1047 (1950).

[4] G. V. Efimov, Commun. Math. Phys. 7, 138 (1968).

[5] G. V. Efimov, Ann. Phys. (N.Y.) 71, 466 (1972).

[6] G. V. Efimov, Int. J. Theor. Phys. 10, 19 (1974).

[7] V. A. Alebastrov and G. V. Efimov, Commun. Math. Phys. 38, 11 (1974).

[8] E. Witten, Nucl. Phys. B268, 253 (1986).

[9] P. H. Frampton and Y. Okada, Phys. Rev. D 37, 3077 (1988).

[10] D. A. Eliezer and R. P. Woodard, Nucl. Phys. B325, 389 (1989).

[11] V. A. Kostelecky and S. Samuel, Phys. Rev. D 42, 1289 (1990).

[12] B. Harms and Y. Leblanc, Ann. Phys. (N.Y.) 242, 265 (1995).

[13] A. A. Tseytlin, Phys. Lett. B 363, 223 (1995).

[14] G. Calcagni and L. Modesto, J. Phys. A 47, 355402 (2014).

[15] G. Calcagni and L. Modesto, Phys. Rev. D 91, 124059 (2015).

[16] S. Hossenfelder, Living Rev. Relativity 16, 2 (2013).

[17] E. Tomboulis, arXiv:hep-th/9702146.

[18] T. Biswas, E. Gerwick, T. Koivisto, and A. Mazumdar, Phys. Rev. Lett. 108, 031101 (2012).

[19] L. Modesto, Phys. Rev. D 86, 044005 (2012).

[20] T. Biswas, A. Conroy, A. S. Koshelev, and A. Mazumdar, Classical Quant. Grav. 31, 015022 (2014).

[21] I. L. Shapiro, Phys. Lett. B 744, 67 (2015).

[22] T. Biswas, T. Koivisto, and A. Mazumdar, J. Cosmol. Astropart. Phys. 11 (2010) 008.

[23] T. Biswas, T. Koivisto, and A. Mazumdar, arXiv:1302.0532.

[24] T. Biswas, A. Mazumdar, and W. Siegel, J. Cosmol. Astropart. Phys. 03 (2006) 009.

[25] A. S. Koshelev, J. High Energy Phys. 10 (2007) 029.
[26] T. Biswas, T. Koivisto, and A. Mazumdar, J. Cosmol. Astropart. Phys. 11 (2010) 008.

[27] L. Modesto, J. W. Moffat, and P. Nicolini, Phys. Lett. B 695, 397 (2011).

[28] A. Conroy, A. Mazumdar, S. Talaganis, and A. Teimouri, Phys. Rev. D 92, 124051 (2015).

[29] L. Modesto and L. Rachwał, Int. J. Mod. Phys. D 26, 1730020 (2017).

[30] L. Buoninfante, A. S. Koshelev, G. Lambiase, and A. Mazumdar, J. Cosmol. Astropart. Phys. 09 (2018) 034.

[31] A. S. Koshelev, J. Marto, and A. Mazumdar, Phys. Rev. D 98, 064023 (2018).

[32] E. Kilicarslan, Turk. J. Phys. 43, 126 (2019).

[33] A. S. Koshelev, J. Marto, and A. Mazumdar, J. Cosmol. Astropart. Phys. 02 (2019) 020.

[34] E. Kilicarslan, Phys. Rev. D 99, 124048 (2019).

[35] 1. de la Cruz-Dombriz, F. J. Maldonado Torralba, and A. Mazumdar, Phys. Rev. D 99, 104021 (2019).

[36] L. Buoninfante and A. Mazumdar, Phys. Rev. D 100, 024031 (2019).

[37] J. Boos, V. P. Frolov, and A. Zelnikov, Phys. Rev. D 97, 084021 (2018).

[38] J. Boos, J. Pinedo Soto, and V. P. Frolov, Phys. Rev. D 101, 124065 (2020).

[39] V. P. Frolov, Phys. Rev. Lett. 115, 051102 (2015).

[40] V. P. Frolov, A. Zelnikov, and T. de Paula Netto, J. High Energy Phys. 06 (2015) 107.

[41] V. P. Frolov and A. Zelnikov, Phys. Rev. D 93, 064048 (2016).

[42] J. Boos, V. P. Frolov, and A. Zelnikov, Phys. Lett. B 782, 688 (2018).

[43] V. P. Frolov and A. Zelnikov, Phys. Rev. D 98, 084035 (2018).

[44] L. Buoninfante, A. Mazumdar, and J. Peng, Phys. Rev. D 100, 104059 (2019). 
[45] V. Arnold, K. Vogtmann, and A. Weinstein, Mathematical Methods of Classical Mechanics (Springer, New York, 2013).

[46] V. Maslov and M. Fedoriuk, Semi-Classical Approximation in Quantum Mechanics, Mathematical Physics and Applied Mathematics (Springer, Netherlands, 1981).
[47] F. Lu, B. Lv, P. Wang, and H. Yang, Nucl. Phys. B937, 502 (2018).

[48] L. D. Landau and E. M. Lifshitz, Quantum Mechanics, 2nd ed. (Pergamon Press, Bristol, 1965). 